\title{
Variations of the solar granulation motions with height using the GOLF/SoHO experiment
}

\author{
S. Lefebvre ${ }^{1}$, R. A. García ${ }^{1}$, S. J. Jiménez-Reyes ${ }^{2}$, S. Turck-Chièze ${ }^{1}$, and S. Mathur ${ }^{1,2}$ \\ 1 Laboratoire AIM, CEA/DSM-CNRS-Université Paris Diderot; CEA, IRFU, SAp, centre de Saclay, 91191 Gif-sur-Yvette, France \\ e-mail: [sandrine.lefebvre;rgarcia@cea.fr] \\ 2 Instituto de Astrofísica de Canarias, 38205 La Laguna, Tenerife, Spain
}

Received 7 June 2008 / Accepted 30 July 2008

ABSTRACT

\begin{abstract}
Context. Below $1 \mathrm{mHz}$, the power spectrum of helioseismic velocity measurements is dominated by the spectrum of convective motions (granulation and supergranulation) and it is difficult to detect the low-order acoustic modes and gravity modes.

Aims. We attempt to understand more clearly the behavior of solar granulation as a function of observing height in the solar atmosphere and with magnetic activity during solar cycle 23 .

Methods. We analyze the Power Spectral Density (PSD) of eleven years of GOLF/SOHO velocity time series data using a Harvey-type model to characterize the properties of the convective motions in the solar oscillation power spectrum. We study the evolution of the granulation with both altitude in the solar atmosphere and solar activity.

Results. We first demonstrate that the traditional use of a Lorentzian profile to describe the envelope of the $p$ modes is unsuitable for GOLF data. To model properly the solar spectrum, we must instead adopt a second Lorentzian profile. Secondly, we show that the granulation clearly evolves with height in the photosphere but does not present any significant variation with the activity cycle.
\end{abstract}

Key words. Sun: activity - Sun: chromosphere - Sun: granulation - Sun: helioseismology - Sun: oscillations - Sun: photosphere

\section{Introduction}

The photosphere, the visible layer of the Sun, is the location where energy transport previously dominated by convection and turbulence is achieved instead by radiation with an optical depth of $2 / 3$. The gas is visible in the form of granules, which penetrate inside the stable photosphere. These granules, as well as other larger structures such as the mesogranules or supergranules, are the manifestation of the different spatial scales of convective motions that occur in this region of the Sun (Zahn 1987; Roudier et al. 1991; Espagnet et al. 1993).

The study of the granulation is particularly important in helioseismology because, on the one hand, it excites the socalled 5-min oscillations, i.e. the acoustic (p) modes, and, on the other hand, it dominates the power spectrum at low frequencies preventing the detection of low-order $\mathrm{p}$ modes. In the case of velocity measurements, the lower detection limit of acoustic modes is established around $1 \mathrm{mHz}$ (García et al. 2001, 2004a; Broomhall et al. 2007). To improve the detection of these modes and to increase the detection probability of gravity modes (Appourchaux et al. 2000; Gabriel et al. 2002; Turck-Chièze et al. 2004; García et al. 2007; Mathur et al. 2007), we need to characterize more precisely the properties of the granulation to reduce, if possible, their impact on the helioseismic measurements. The coming GOLF-NG instrument will soon address this problem (Turck-Chièze et al. 2006). This new-generation instrument should improve the signal-to-noise ratio $(\mathrm{S} / \mathrm{N})$ of lowfrequency modes by measuring the Doppler velocity at different heights in the solar atmosphere. It would therefore benefit from the reduction in the coherence of the granulation with the atmospheric altitude (García et al. 2004b).
In this paper, we analyze the Power Spectral Density (PSD) of velocity time sub-series from the GOLF ${ }^{1}$ instrument onboard $\mathrm{SOHO}^{2}$, which is a solar disk integrated resonant spectrometer. Using this instrument, we can study the mean behavior of the solar granulation in parts of the atmosphere. This work complements the study of Espagnet et al. (1995), in which it was found that the photosphere is highly structured with two distinct layers below and above about $90 \mathrm{~km}$. With GOLF, and the technique used in Jiménez-Reyes et al. (2007), we are able to study, without the need of exceptional spatial resolution, a region located between 250 and $550 \mathrm{~km}$ above the photosphere. We show that the granulation evolves with height in the photosphere and that the granules tend to have shorter lifetimes with a weaker velocity when higher up in the atmosphere.

Section 2 is devoted to the data analysis, with first a brief summary of the velocity calibration procedure of the GOLF signal and secondly a description of the fitting procedure of the power spectra in more detail. Section 3 is dedicated to a detailed study of the granulation motions and its evolution with time during the solar cycle. Finally, in Sect. 4, we emphasize the main results of this paper and anticipate forthcomong research.

\section{Data analysis}

\subsection{Data}

GOLF is a resonant scattering spectrophotometer that measures the Doppler shift of the neutral sodium doublet $\left(D_{1}\right.$ at $\lambda=$ $589.6 \mathrm{~nm}$, and $D_{2}$ at $\left.\lambda=589.0 \mathrm{~nm}\right)$.

\footnotetext{
${ }^{1}$ Global Oscillation at Low Frequencies (Gabriel et al. 1995).

2 SOlar and Heliospheric Observatory (Domingo et al. 1995).
} 
In the present study, we use time series data of this experiment calibrated in terms of velocity (García et al. 2005). In this instrument, the light originating in the solar sodium absorption line (half-width $500 \mathrm{~m} \AA$ ) traverses a sodium vapour cell, placed in a longitudinal magnetic field of $\sim 5000$ Gauss, where it is absorbed and re-emitted in all directions. This scattered light is split symmetrically into its Zeeman components allowing a measurement on either side of the wings of the solar absorption profile. The scattered photons are collected by two photomultiplier tubes. Due to a malfunction in the polarization subsystem that switches between the two wings of the sodium doublet, and to ensure a $100 \%$ duty cycle, a single-wing working cycle has been selected since April 11, 1996. The operation of the instrument can be divided into three distinct time periods: between April 1996 and June 25, 1998, GOLF observed the blue wing of the sodium profile; after the SoHO recovery mission in September 1998, GOLF restarted by observing the red wing until November 18, 2002, when it reverted again to observing the blue-wing configuration. Since then, it has been operating, unchanged, in this configuration.

The main velocity field $\left( \pm 0.5 \mathrm{~km} \mathrm{~s}^{-1}\right)$ measured by GOLF is the SoHO orbital velocity. Due to this movement, the working points on the sodium profile vary with a 1 -year periodicity. GOLF therefore analyzes different altitudes in the solar atmosphere (see Fig. 1). As the orbital velocity $V_{\text {orb }}$ increases, the measurements taken on the blue wing are closer to the photosphere, whereas on the red wing the measurements are further from the photosphere (García et al. 2004b).

The transformation from the observed counting rates to an intensity-like variable suitable for a velocity scaling includes two major steps, which are, firstly, corrections to the raw measurements to remove instrumental effects, and secondly, compensation for the Sun-spacecraft variable distance. Before calibrating the data into velocity, corrections of some instrumental effects are applied to the counting rates including those applied to the photomultipliers, such as dead time, aging of the phototubes, and high voltage perturbations, as well as sodium cell stem, photocathodes, and filter temperature corrections (see García et al. 2005 , for further details). In particular, in the second period of the blue-wing measurements, the correction of the photomultipliers temperature was no longer valid and it was removed from the calibration pipeline. As a consequence, there is a small noncorrected offset in this final segment of data compared to the first period. Finally, the calibration introduces a smooth high-pass filter with a cut-off frequency of about $1 \times 10^{-6} \mathrm{~Hz}$ that filters out all the frequencies below that one. To confirm the independence of our results from the calibration procedure, we repeated the analysis using another velocity calibration procedure developed by Ulrich et al. (2000), which involves a different set of corrections. The results were qualitatively similar for both calibration methods.

GOLF has operated for almost 4200 days, a long period of time that allows a detailed study of the temporal evolution of its power spectrum during the entire solar cycle 23 . To understand this and, in particular, the granulation, we use another two quantities:

- The MPSI (Magnetic Plage Strength Index), developed by Chapman \& Boyden (1986), Ulrich (1991), and Parker et al. (1998) that represents a proxy of the magnetic activity. This index is determined from the 150 -foot solar tower magnetograms by measuring the sum (in all pixels) of the absolute value of the magnetic field strengths between 10 and 100 Gauss. This number is then divided by the total number

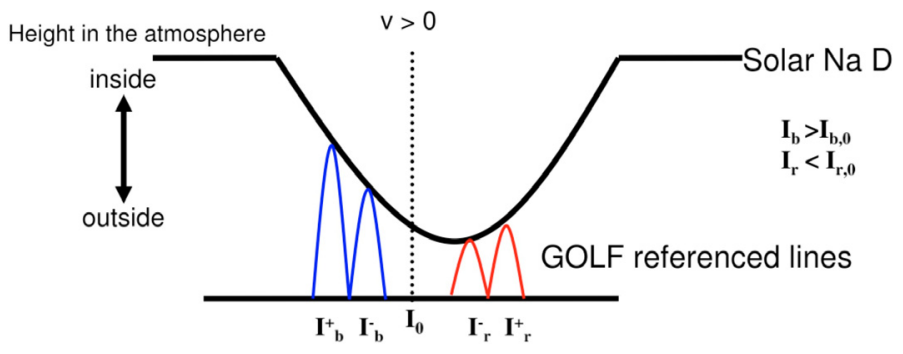

Fig. 1. Representation of the GOLF measurements. The Solar Na D line is displaced from its rest position due to a positive velocity $(V>0)$. The BW (blue wing) probes the line profile closer to its continuum level, which is deeper (close to the photosphere) in the solar atmosphere. The RW (red wing) studies the part of the profile closer to the bottom of the line, i.e. further up into the solar atmosphere.

of pixels (regardless of magnetic field strength) in the magnetogram ${ }^{3}$.

- The GOLF observational height in the solar atmosphere computed from the line-of-sight velocity taking into account the main displacement of the $\mathrm{Na}$ lines from the rest position. We use the procedure described in Jiménez-Reyes et al. (2007) to translate these velocities into a value of height in the solar atmosphere.

It is important to remember that GOLF measures Doppler shifts in the Na line. It is therefore mostly sensitive to a mixture of the vertical velocity towards the disk center and the horizontal velocity towards the solar limb. Due to the single-wing configuration, the highest sensitivity will not be at the disk center but shifted towards the East or the West depending on the wing where the measurements are completed (see Fig. 3 of García et al. 1998).

\subsection{Fitting procedure}

The original GOLF time series are sampled every $20 \mathrm{~s}$ and we used series of 91.25 days shifted every 22.81 days. It is important to note that in the statistical analysis, we use independent subseries only, while in the figures we will plot all of them. This length of data was chosen to be a good trade-off between the frequency resolution and the number of independent series needed to track correctly the variations in the height of the atmosphere during a year. Fast Fourier Transform (FFT) techniques were used to derive each individual spectrum. The normalization used in the PSD is the so-called "one-sided" power spectral density (Press et al. 1992). A first selection over the spectra was made. We considered only spectra with a duty cycle above $85 \%$ and eliminated 3 series in September, October and November of 2002 - just prior to the return to the observing of the blue wing because they contained a mixture of both wings.

The velocity power spectral density (PSD) can be described by a model in which each source of solar convective motions is described by an empirical law initially proposed by Harvey (Harvey 1985; Harvey et al. 1993). This model corresponds to an exponentially decaying time function, plus one or two Lorentzian functions for the envelope of $p$-modes

${ }^{3}$ See http://www.astro.ucla.edu/ obs/150_data.html 
(Vásquez Ramió et al. 2002) and a constant for the photon noise. The function fitted to the spectra, including both non-periodic and periodic components, is therefore given by:

$$
\begin{aligned}
P(v)= & N_{\mathrm{ph}}+\sum_{i=1}^{N} \frac{4 \sigma_{i}^{2} \tau_{i}}{1+\left(2 \pi v \tau_{i}\right)^{b_{i}}} \\
& +\sum_{j=1}^{M} A_{j}\left[\frac{\Gamma_{j}^{2}}{\left(v-v_{0_{j}}\right)^{2}+\Gamma_{j}^{2}}\right]^{c_{j}}
\end{aligned}
$$

where

- $P(v)$ is the power spectral density;

- $N_{\mathrm{ph}}$ is the photon noise in units of $(\mathrm{m} / \mathrm{s})^{2} / \mathrm{Hz}$;

- $i$ corresponds to the non-periodic velocity fields;

- $j$ corresponds to the periodic components;

$-\sigma_{i}$ and $\tau_{i}$ are the rms-velocity $(\mathrm{m} / \mathrm{s})$ and the characteristic time (s) of the $i$ th background component, respectively (the limit of the first sum $N$ varies depending on the number of non-periodic background components of the spectrum to be fitted);

- $A_{j}$ and $v_{0_{j}}$ are the power $\left((\mathrm{m} / \mathrm{s})^{2} / \mathrm{Hz}\right)$ and the central frequency $(\mathrm{Hz})$ of the Lorentzian profiles to be fitted to the periodic components in the higher frequency region of the spectrum, while $\Gamma_{j}$ determines its width $(\mathrm{Hz})$. The $M$ possible peaks to be fitted can be identified with the so-called photospheric or/and the chromospheric component;

- and finally, $c_{j}$ (as well as $b_{i}$ ) are decay rates.

Before fitting the spectra, due to a significant number of bins and because the points are compressed to a logarithmic scale at high frequencies, different local averages were calculated until the data were equally spaced in a logarithmic scale. This allowed us to fit the model along the entire frequency axis. The fitting was performed in the logarithmic space of the PSD by employing a standard non-linear least-square method (LevensbergMarquardt; Press et al. 1992).

Two non-periodic components were fitted for granulation and supergranulation with $b_{i}=2$. To fit correctly the $p$-mode envelope, it was necessary to use 2 Lorentzian profiles. In preliminary fits, $\Gamma_{j}$ was obtained close to $1 \times 10^{-3}$, then we fixed the coefficient to this value in the remainder of the fits. In previous work (Régulo et al. 2002), an additional factor $\left(v / v_{0_{j}}\right)^{a_{j}}$ was found in the Lorentzian term, but following these authors, we decided to fix the coefficient $a_{j}$ of this term to zero in our fits.

We attempted to isolate the different effects in the background spectrum that were merged in the data: the dependence with solar activity and the dependence with observing point in both wings (solar atmospheric depth). Which effect was dominant? In the following, we noted BW1, the first period in the blue wing, RW, the following red-wing configuration, and BW2, the second period in the blue-wing mode. The fits had 11 coefficients, i.e. 2 coefficients for the granulation and supergranulation, 3 coefficients for each of both of the Lorentzians used to fit the $p$-mode envelope (the $\Gamma$ factor being fixed), plus the photon noise. If we fitted only 8 coefficients, only one Lorentzian is used for the acoustic-mode envelope.

Figure 2 shows an example of a spectrum in which each component is represented by a different curve and the sum of these curves represents the total fit (left-hand panels). The ratio between the PSD and the fit is also shown (right-hand panels). We can see the difference between a fit with one Lorentzian (8 parameters, top panels) and a fit with two Lorentzians (11 parameters, bottom panels) corresponding to the adjustment of the $p$-mode envelope. These plots indicate the level of the requirement that both Lorentzians adjust the $p$-mode envelope correctly and decrease the amplitude of both cases excess power present close to 0.003 and $0.005 \mathrm{~Hz}$ in the 8-parameter fit. In the following section, we focus on a thorough analysis of the granulation noise behavior. To do so, we model the PSD using a fit with two Lorentzian profiles; the conclusions obtained for this convective pattern would however be qualitatively the same when using a fit with a single Lorentzian. The analysis of other parameters will be considered in future work. However, we note that the supergranulation fit is less robust than the granulation fit. The supergranulation region is certainly affected by the calibration of the data that filters this region slightly (García et al. 2005), and by the small number of points in this region due to a small frequency resolution. If we had used non-filtered series of 365 days, the fit of the supergranulation would have been more stable.

\section{Results}

\subsection{Evolution of the granulation with time}

Figure 3 shows the temporal evolution of the fitting parameters for the granulation as well as the activity during the last solar cycle represented by the MPSI index. The standard deviation velocity in each wing is different with a lower value in the red wing. The velocity is higher in BW2 than BW1 as a consequence of the change in the calibration of the second blue-wing period when the photocathode temperature correction were removed. We clearly see the signal dependence with orbital velocity as a one-year modulation, enabling us to probe different heights in the atmosphere as we will see in the next section. This oneyear modulation has an opposite phase between the blue and red wings, which can be clearly seen in the top panels. The blue wing is directly correlated with the orbital velocity (third panel), while the red wing is anticorrelated. The amplitude is larger in the red wing than in the blue one.

\subsection{Evolution of the granulation with the height and the solar activity}

The height in the photosphere is computed following the procedure described in detail in Jiménez-Reyes et al. (2007), and briefly summarized here:

- the so-called response functions (hereafter RFs; see Mein 1971; and Beckers \& Milkey 1975) of the Na D1 and D2 Fraunhofer lines are computed: these functions measure the reaction of the line profile when the atmosphere is perturbed locally at a given height. The quiet Sun model C (Vernazza et al. 1981) was employed as a reference because it produces spectral line profiles (both photospheric and chromospheric) that are generally in good agreement with observations made at low spatial resolution;

- for each line, we computed the RFs for changes in velocity by applying small perturbations in the model at each optical depth;

- the RFs for the full line profiles were then convolved with the wavelength filter response of the instrument;

- the calculations considered changes in the line-of-sight velocity at different times of year.

Figure 4 shows the evolution of the granulation parameters the altitude in the solar atmosphere and the MPSI index. To provide a quantitative assessment of the observed changes in the velocity 



Fig. 2. Results of two different type of fits, as explained in the text, applied to a GOLF spectrum of an arbitrary taken subseries of 91.25 days long. Top: left, PSD with a fit using 8 parameters (one lorentzian) to adjust the $p$-mode envelope; right, ratio between the PSD and the fit around the envelope of $p$-modes. Bottom: left, PSD with a fit using 11 parameters (two lorentzians) to adjust the $p$-mode envelope; right, ratio between the PSD and the fit around the envelope of $p$-modes. The dashed lines represent the limits inside which the fit is performed. The color used for the different fits are: gray for the super granulation contribution, magenta for the granulation contribution, blue for the $p$-mode envelope, green for the noise and red for the harvey function (the sum of the granulation and supergranulation contributions).

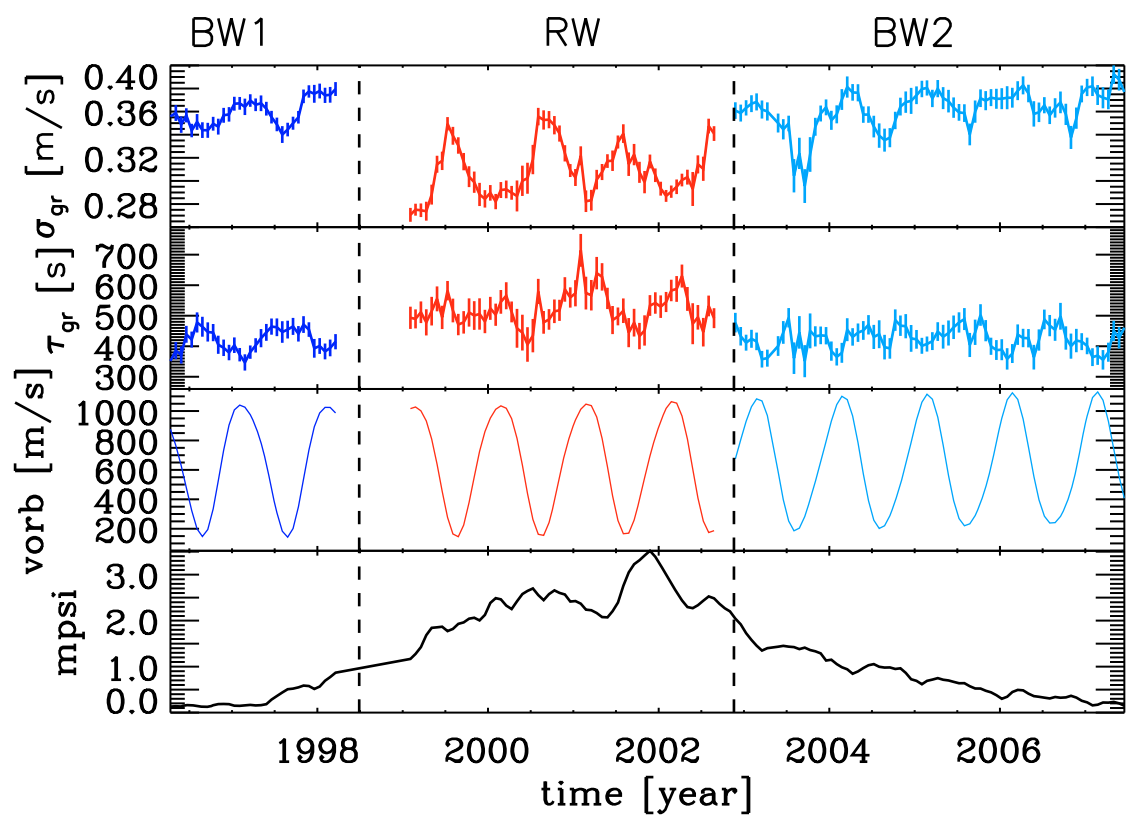

Fig. 3. Temporal evolution of the granulation $(\sigma$ and $\tau$, top and second panel respectively) of the GOLF PSD during the solar cycle represented by the MPSI index plotted in the bottom panel. The third panel represent the orbital velocity with time. Here, the vertical dotted lines indicate the date of the changes in the GOLF observing configuration. 



Fig. 4. Evolution of the granulation velocity and characteristic time versus the height (left) and the MPSI index (right). The linear trend with MPSI has been computed after removing the trend with height. + and $\square$ : respectively BW1 and BW2; $\triangle$ : RW. The solid lines in each panel are the best-fit linear regression relation for independent series and for the separate blue- and red-wing data sets. We note the jump between red and blue operating mode. All panels show overlapping data for illustrative purposes, with errors plotted only on the independant data.

and characteristic time, we performed a linear regression with each of the parameters: the characteristic of each linear trend is summarized in Table 1 in terms of the variation with height and in Table 2 for the variation with the MPSI index. We used data from independent series that did not overlap in time. This was achieved for each of the blue- and red-wing periods following the same method as in Jiménez-Reyes et al. (2007). For the evolution with the MPSI index, the trend was calculated after removing the trend with height. Errors in the general fit were used to assess the quality of the linear fitting. For clarity, only error bars for the independent series are plotted. The best fit of each of the red and blue data sets are indicated by the black lines in the panels of Fig. 4. We also computed the Pearson product-moment and Spearman's rank correlation coefficients for data presented in both tables. In Table 1, there are also coefficients for the linear regression relation fitted to all wings. For the velocity, to remove the offset, we applied an adhoc correction by adding (or removing) half of the difference in the velocity intercepts i.e. $(0.48-0.44) / 2$ to BW1 (or from BW2).

The dependence on depth in the solar atmosphere can be clearly seen. As explained above, when the observations are completed for the red wing, we are observing higher in the solar atmosphere than when the blue wing is used. We notice several interesting behaviours:

- for each wing, there is a clear dependence on height: a decrease in $\sigma_{\mathrm{gr}}$ and an increase in $\tau_{\mathrm{gr}}$ with altitude in the photosphere, which can be interpreted as a longer lifetime for granules higher up in the atmosphere. There is a strong correlation with height, more importantly for $\sigma_{\mathrm{gr}}$ than for $\tau_{\mathrm{gr}}$ (gradients fitted are more significant at 4.4, 10.6, 4.7 $\sigma$ and $2.8,2.6,1.8 \sigma$, respectively, for each mode operation). For $\sigma_{\mathrm{gr}}$, the difference in the intercept between each wing is significant, whereas the error bars of the intercepts for $\tau_{\text {gr }}$ are too significant for the measurements to be important. It is worth noting that the significance of the linear fit for $\tau_{\mathrm{gr}}$ is more important when considering all confused wings;

- concerning the velocity, there is an offset between the measurements for the two blue wings but the same slope and same linear trend (slope and offset) for the characteristic time; this is normal because a difference in the temperature calibration between the two wings can affect $\sigma_{\mathrm{gr}}$ but not the value of $\tau_{\mathrm{gr}}$. The offset in velocity is significant and certainly due to the difference in calibration;

- the difference in slope of the velocity between the blue wing and the red wing is weak. It appears that there is a slight break in the profile of $\sigma_{\mathrm{gr}}$ with height close to $400 \mathrm{~km}$;

- however, the variation with the MPSI is insignificant: the scatter in the residuals with activity is too large.

\section{Conclusion and perspectives}

In this paper, we have investigated the vertical structure and time evolution of the solar granulation by means of a novel methodology based on the analysis of the full-disk Sun-as-a-star Doppler velocity observations. We have therefore been able to study the vertical velocity fluctuations and lifetimes of the solar granulation. We have shown that the GOLF PSD can be characterized 
Table 1. Linear regression analysis results for the granulation with height.

\begin{tabular}{cccccccccc}
\hline \hline Epoch & Parameter & Intercept & Gradient & $\begin{array}{c}\text { Significance } \\
\text { of fitted gradient }\end{array}$ & $\chi^{2}$ & $N$ & $r_{\mathrm{P}}$ & $r_{\mathrm{S}}$ & $P_{\mathrm{S}}$ \\
\hline BW1 & $\tau_{\mathrm{gr}}[\mathrm{s}]$ & $125 \pm 102$ & $0.91 \pm 0.32$ & $2.8 \sigma$ & 1.66 & 8 & 0.71 & 0.55 & $1.60 \times 10^{-1}$ \\
& $\sigma_{\mathrm{gr}}[\mathrm{m} / \mathrm{s}]$ & $0.44 \pm 0.02$ & $(-3.06 \pm 0.70) \times 10^{-4}$ & $4.4 \sigma$ & 1.99 & 8 & 0.79 & 0.85 & $6.53 \times 10^{-3}$ \\
$\mathrm{RW}$ & $\tau_{\mathrm{gr}}[\mathrm{s}]$ & $238 \pm 109$ & $0.59 \pm 0.23$ & $2.6 \sigma$ & 0.94 & 15 & 0.62 & 0.64 & $1.03 \times 10^{-2}$ \\
& $\sigma_{\mathrm{gr}}[\mathrm{m} / \mathrm{s}]$ & $0.53 \pm 0.02$ & $(-4.54 \pm 0.43) \times 10^{-4}$ & $10.6 \sigma$ & 2.43 & 15 & 0.83 & 0.79 & $4.22 \times 10^{-4}$ \\
$\mathrm{BW} 2$ & $\tau_{\mathrm{gr}}[\mathrm{s}]$ & $272 \pm 82$ & $0.46 \pm 0.26$ & $1.8 \sigma$ & 0.81 & 19 & 0.39 & 0.25 & $3.04 \times 10^{-1}$ \\
& $\sigma_{\mathrm{gr}}[\mathrm{m} / \mathrm{s}]$ & $0.48 \pm 0.02$ & $(-3.07 \pm 0.66) \times 10^{-4}$ & $4.7 \sigma$ & 1.50 & 19 & 0.70 & 0.63 & $4.12 \times 10^{-3}$ \\
All wings & $\tau_{\mathrm{gr}}[\mathrm{s}]$ & $214 \pm 24$ & $0.64 \pm 0.07$ & $9.1 \sigma$ & 0.93 & 42 & 0.84 & 0.78 & $1.49 \times 10^{-9}$ \\
& $\sigma_{\mathrm{gr}}[\mathrm{m} / \mathrm{s}]$ & $0.47 \pm 0.005$ & $(-3.34 \pm 0.13) \times 10^{-4}$ & $25.7 \sigma$ & 1.95 & 42 & 0.93 & 0.88 & $4.59 \times 10^{-15}$ \\
\hline
\end{tabular}

Note. The seventh column lists the numbers of independent data points $N$ employed in each of the regression analysis. The three last columns show the correlation between the linear regression and the data: $r_{\mathrm{P}}$, Pearson product-moment correlation coefficient; $r_{\mathrm{S}}$, Spearman's rank correlation coefficient; and $P_{\mathrm{S}}$, two-sided probability of Spearman coefficient.

Table 2. Linear regression analysis results for the granulation (residuals after removing the trend with height) with MPSI index.

\begin{tabular}{|c|c|c|c|c|c|c|c|c|c|}
\hline Epoch & Parameter & Intercept & Gradient & $\begin{array}{c}\text { Significance } \\
\text { of fitted gradient }\end{array}$ & $\chi^{2}$ & $N$ & $r_{\mathrm{P}}$ & $r_{\mathrm{S}}$ & $P_{\mathrm{S}}$ \\
\hline \multirow[t]{2}{*}{ BW1 } & & & 72 & $18 \sigma$ & 1.15 & 8 & 022 & 0.19 & $6.51 \times 10^{-1}$ \\
\hline & $\sigma[\mathrm{m} / \mathrm{s}]$ & $(-7.88 \pm 4.09) \times 10^{-3}$ & $(2.25 \pm 0.97) \times 10^{-2}$ & & & 8 & 0.5 & 0.60 & $10^{-1}$ \\
\hline \multirow[t]{2}{*}{ RW } & $\tau[\mathrm{s}]$ & $-49 \pm 43$ & $21 \pm 18$ & 1 & 0 & 15 & 0.04 & -0.02 & $9.50 \times 10^{-1}$ \\
\hline & $\sigma[\mathrm{m} / \mathrm{s}]$ & $(-19.14 \pm 7.39) \times 10^{-3}$ & $(0.82 \pm 0.31) \times 10^{-2}$ & 2. & 1 & 1 & 0. & 0.24 & $3.98 \times 10^{-1}$ \\
\hline \multirow[t]{2}{*}{ BW2 } & $\tau[s]$ & $-25 \pm 15$ & $20 \pm 15$ & $1.3 \sigma$ & 0.58 & 19 & 0.45 & 0.32 & $1.88 \times 10^{-1}$ \\
\hline & $\sigma[\mathrm{m} / \mathrm{s}]$ & $(7.96 \pm 3.76) \times 10^{-3}$ & $(-9.16 \pm 3.71) \times 10^{-3}$ & $2.5 \sigma$ & 1.14 & 19 & 0.38 & 0.41 & $8.37 \times 10^{-2}$ \\
\hline
\end{tabular}

Note. The seventh column lists the numbers of independent data points $N$ employed in each of the regression analysis. The three last columns show the correlation between the linear regression and the data: $r_{\mathrm{P}}$, Pearson product-moment correlation coefficient; $r_{\mathrm{S}}$, Spearman's rank correlation coefficient; and $P_{\mathrm{S}}$, two-sided probability of Spearman coefficient.

correctly by our model, which is a Harvey function with two Lorentzian profiles.

This work extends the study of Espagnet et al. (1995), which found that the photosphere is highly structured with two distinct layers below and above about $90 \mathrm{~km}$. With GOLF, we studied the photosphere above $\approx 280 \mathrm{~km}$ and showed that granules tend to live longer with a weaker velocity when higher up in the atmosphere. Following the results of Title et al. (1989), we found that there was a strong correlation between the granule sizes and lifetime. Therefore, we were able to conclude that larger granules reach closer to the top of the photosphere, while the penetrating ability of granules decreases in general with decreasing size. Figure 4 illustrates results in the case of a lifetime of about 400-550 s, for granules between 250 and $550 \mathrm{~km}$. From Title et al. (1989) and their Fig. 21, we can estimate that the granules at these altitudes have a lifetime-average size of about 1.2 arcsec and a maximum size of about 1.4-1.5 arcsec.

We have found that the granulation rms velocities $\left(\sigma_{\mathrm{gr}}\right)$ are between 0.28 and $0.4 \mathrm{~m} \mathrm{~s}^{-1}$. These values differ from those obtained from high-resolution measurements, which are about $1 \mathrm{~km} \mathrm{~s}^{-1}$. The difference in spatial resolution is the most likely cause of the difference of three orders of magnitude.

However, we were unable to detect any change with solar activity. This is consistent with the result of Jiménez-Reyes et al. (2003) that the solar cycle effects are small compared to the change in observing height in the photosphere, due to the orbital motion. It could be due to the fact that GOLF observes the more stable part of the sodium lines. However, if we have not found any significant variation with cycle, another study (Muller et al. 2007) found a cyclic variation in the contrast of the granules, almost in phase with the solar cycle, for which the contrast was smaller at the periods of solar maximum, but no corresponding variation in the length scale.

This work enlightens two main areas of research to be explored in the future: (1) to improve our understanding of the evolution of the entire solar convective background during the activity cycle, and, in particular, the evolution of the acoustic-mode envelope characterized by the presence of two Lorentzians. The excess power characterized by the second Lorentzian probably corresponds to the presence of chromospheric modes; (2) to extend this study to our new instrument GOLF-NG observation. This instrument will monitor a larger part of the solar atmosphere by completing the 8 extractions of the Doppler velocity with a proper determination of their location due to the measurement of both wings and the use of only the D1 line (Jiménez-Reyes et al. 2007; Turck-Chièze et al. 2006; TurckChièze et al. 2008).

Acknowledgements. The authors thank all their colleagues (scientists, engineers and technicians) involved with the GOLF instrument aboard SoHO which is a space mission of international cooperation between ESA and NASA. S. Lefebvre is supported by a CNES/GOLF research engineer contract in SAp. S. J. Jiménez-Reyes acknowledge partial financial support from Spanish grants PNAyA2007-62651 and the support of European Helio- and Asteroseismology Network (HELAS), a major international collaboration funded by the European Commission's Sixt Framework Programme.

\section{References}

Appourchaux, T., Fröhlich, C., Andersen, B., et al. 2000, ApJ, 538, 401 Beckers, J. M., \& Milkey, R. W. 1975, Sol. Phys., 43, 289

Broomhall, A. M., Chaplin, W. J., Elsworth, Y., \& Appourchaux, T. 2007, MNRAS, 379, 2

Chapman, G. A., \& Boyden, J. E. 1986, ApJ, 302, L71 
Domingo, V., Fleck, B., \& Poland, A. I. 1995, Sol. Phys., 162, 1

Espagnet, O., Muller, R., Roudier, T., \& Mein, N. 1993, A\&A, 271, 589

Espagnet, O., Muller, R., Roudier, T., Mein, N., \& Mein, P. 1995, A\&AS, 109, 79

Gabriel, A. H., Grec, G., Charra, J., et al. 1995, Sol. Phys., 162, 61

Gabriel, A. H., Baudin, F., Boumier, P., et al. 2002, A\&A, 390, 1119

García, R. A., Roca Cortés, T., \& Régulo, C. 1998, A\&AS, 128, 389

García, R. A., Régulo, C., Turck-Chièze, S., et al. 2001, Sol. Phys., 200, 361

García, R. A., Corbard, T., Chaplin, W. J., et al. 2004a, Sol. Phys., 220, 269

García, R. A., Jiménez-Reyes, S. J., Turck-Chièze, S., \& Mathur, S. 2004b, in SOHO 14 Helio- and Asteroseismology: Towards a Golden Future, ed. D. Danesy, ESA Special Publication, 559, 432

García, R. A., Turck-Chièze, S., Boumier, P., et al. 2005, A\&A, 442, 385

García, R. A., Turck-Chièze, S., Jiménez-Reyes, S. J., et al. 2007, Science, 316, 1591

Harvey, J. W. 1985, in Probing the depths of a Star: the study of solar oscillations from space, ed. R. W. Noyes, \& E. J. Rhodes, JPL, 400, 327

Harvey, J. W., Duvall, Jr., T. L., Jefferies, S. M., \& Pomerantz, M. A. 1993, in Seismic Investigation of the Sun and Stars, ed. T. M. Brown, ASP Conf. Ser., 42, GONG 1992, 111

Jiménez-Reyes, S. J., García, R. A., Jiménez, A., \& Chaplin, W. J. 2003, ApJ, 595,446

Jiménez-Reyes, S. J., Chaplin, W. J., Elsworth, Y., et al. 2007, ApJ, 654, 1135
Mathur, S., Turck-Chièze, S., Couvidat, S., \& García, R. A. 2007, ApJ, 668, 594 Mein, P. 1971, Sol. Phys., 20, 3

Muller, R., Hanslmeier, A., \& Saldaña-Muñoz, M. 2007, A\&A, 475, 717

Parker, D. G., Ulrich, R. K., \& Pap, J. M. 1998, Sol. Phys., 177, 229

Press, W. H., Teukolsky, S. A., Vetterling, W. T., \& Flannery, B. P. 1992, Numerical recipes in FORTRAN, The art of scientific computing, 2nd ed. (Cambridge: University Press)

Régulo, C., Roca Cortés, T., \& Vázquez Ramió, H. 2002, in Solar Variability: From Core to Outer Frontiers, ed. J. Kuijpers, ESA Special Publication, 506, 889

Roudier, T., Vigneau, J., Espagnet, O., et al. 1991, A\&A, 248, 245

Title, A. M., Tarbell, T. D., Topka, K. P., et al. 1989, ApJ, 336, 475

Turck-Chièze, S., García, R. A., Couvidat, S., et al. 2004, ApJ, 604, 455

Turck-Chièze, S., Carton, P.-H., Ballot, J., et al. 2006, Adv. Space Res., 38, 1812

Turck-Chièze, S., Carton, P.-H., Mathur, S., et al. 2008, Astron. Nachr., 329, 521 Ulrich, R. K. 1991, Adv. Space Res., 11, 217

Ulrich, R. K., García, R. A., Robillot, J.-M., et al. 2000, A\&A, 364, 799

Vásquez Ramió, H., Roca Cortés, T., \& Régulo, C. 2002, in Solar Variability: From Core to Outer Frontiers, ed. J. Kuijpers, ESA Special Publication, 506, 897

Vernazza, J. E., Avrett, E. H., \& Loeser, R. 1981, ApJS, 45, 635

Zahn, J.-P. 1987, in Solar and Stellar Physics, ed. E.-H. Schröter, \& M. Schüssler, Lect. Notes Phys. (Berlin: Springer Verlag), 292, 55 